\title{
Lockdown - a time for all professionals working with children to act
}

The current SARS-CoV-2 pandemic has resulted in dramatic changes in the way of life of children and their caregivers, even though children are the least likely to be infected by the novel coronavirus and, if infected, are likely to have the lowest risk of mortality. In South Africa (SA), the effects of COVID-19 have been most evident in the poorest sectors of our population, although not sparing children in the suburbs. The severity of the lockdown has resulted in mass disruption of the informal economy in particular, with dramatic effects on the earning capacity of families in townships, the disruption of extended family networks, childcare facilities, schooling, healthcare and nutritional support, among many other possible negative effects. Although there is consensus among healthcare professionals and those in the social services that the above description of the effects of COVID-19 on the lives of children is a fair reflection of the actual situation, the picture has been largely built up from anecdotal reports. However, there are little hard data to substantiate the magnitude of the unintended consequences of lockdown (with its sometimes nonsensical regulations) and, on occasion, its draconian enforcement on the physical and psychological wellbeing of children in SA.

As healthcare professionals, we should make every effort to document the fall-out from SA's attempt to control the spread of COVID-19 across the country - not only to draw the attention of health authorities to areas needing immediate redress, but also to highlight the possible long term consequences of the disrupted economic, educational, health and family structures on the wellbeing of children. To obtain an overall SA perspective, there is a need for collaborative studies across the provinces, including urban and rural regions. Particular attention should be paid to children living in informal settlements and those whose parents are undocumented immigrants in SA, as these are likely to be the most affected by the lockdown. Of great concern is the effect of the escalating unemployment rates on the nutritional status of children (in particular acute wasting), the disruption of health services on immunisation rates among young children and on the compliance of the treatment of chronic diseases such as diabetes, tuberculosis and HIV, as well as the disruption of schooling on education. Equally concerning is the anecdotal increase in family violence, which has occurred since lockdown.

What could be an appropriate research response? Of critical importance at this time is that any data collected should be as accurate and complete as possible, no matter where it is collected, and preferably should be recorded across a number of centres to provide a broad picture of what is happening. In the immediate throes of the COVID-19 pandemic, there is merit in conducting simple epidemiological studies. A laudable example is the National Institute of Communicable Diseases' DATCOV19 sentinel surveillance system monitoring COVID-19 related hospitalisations at 418 SA private and public hospitals. Its identification of the low proportion (1.4\%) of children in the 1 - 9-year age group among the total SARS-CoV-2 positive tests from hospitals, for instance, offers some reassurance that local disease acquisition patterns in children are mirroring those seen in high incidence COVID-19 countries. These data assist in motivating for actions that return children to normal living, such as the reopening of schools.

In hospital and clinic settings, description of the incidence of conditions such as severe acute malnutrition, vaccine preventable diseases and child non-accidental physical and sexual injury in the period preceding, during, and post-lockdown may well confirm the anticipated negative effects of the pandemic on child nutrition, health-seeking behaviour and abuse - well before mortality data reveal the true devastation of the pandemic months later. The benefits of this type of research would be to force a more urgent and substantive response from the government and the NGO sector to nutrition and essential service provision than is currently the case. Similarly, immediate monitoring and description of COVID19 infection and its interaction with other co-morbidities and vulnerabilities, disaggregating these by age, sex, and geography, could help craft appropriate health system responses. The pooling of data from hospitals countrywide could accelerate the recognition of adverse effects.

The potential research agenda spawned by the COVID-19 pandemic's impact on children is vast, extending from children's mental health to educational outcomes and matters of social protection. Some questions have a clear medical basis and are of vital public health importance, such as the effects of school and childcare centre reopening on child and adult COVID-19 infection rates. Others require a cross-disciplinary approach. All require a willingness by child health practitioners, including registrars, fellows, and Master's students to venture beyond their traditional comfort zones of hospital-based, quantitative studies to engaging with other more pertinent research themes and embracing the challenges posed by the new knowledge gap carved by COVID- 19 .

Now is the time for us as child health professionals to focus our research efforts on understanding the consequences of the COVID-19 pandemic on the health and wellbeing of SA's children and on putting forward appropriate strategies to address these.

Haroon Saloojee, MB BCh, MSc (Med), FCPaed (SA)

Professor and Head: Division of Community Paediatrics, Department of Paediatrics and Child Health, Faculty of Health Sciences, University of the Witwatersrand, Johannesburg, South Africa

Haroon.Saloojee@wits.ac.za

John M Pettifor, MB BCh, PhD (Med), FCPaed (SA), MASSAf, DSc (Med) (hon causa)

Editor-in-Chief and Emeritus Professor, Department of Paediatrics and Child Health, Faculty of Health Sciences,

University of the Witwatersrand, Johannesburg, South Africa John.Pettifor@wits.ac.za 